\title{
A Microscopic View on Aerosols
}

\author{
$\underline{\text { Anders Brostrøm }}^{1}$ and Kristian Mølhave ${ }^{1}$
}

${ }^{1}$ Technical University of Denmark, DTU Nanolab, Lyngby, Denmark.

Atmospheric aerosol particles have a major influence on climate both through direct interaction with sunlight and their role in cloud formation and growth[1,2]. The fate of particles in the atmosphere are governed by their physical and chemical properties. However, these properties are highly complex, as particles can vary individually in size, shape, composition, and physical state. The properties may even change over time due to evaporation, condensation, and oxidation processes caused by a constant equilibrium with the ever changing atmospheric environment. Due to this complexity, aerosol particles are among the major uncertainties in current climate models and predictions of global warming[3].

Aerosol particles have been studied intensely in the past, but mainly by real-time instruments, which assume spherical and homogeneous particles, thus excluding potentially crucial parameters such as shape and physical state e.g. solid, liquid, phase separated, or glassy[4]. Here we demonstrate the use of in situ electron microscopy to visualize and quantify aerosol condensation processes under atmospheric conditions.

We spray atmospherically relevant particles directly onto Si chips with electron transparent $\mathrm{SiN}_{\mathrm{x}}$ windows. By fitting the chips in an in situ TEM holder we can image the particles in a controlled atmospheric environment inside the microscope. In addition, we can flow gas of known composition through the holder, enabling us to visualize condensation or oxidation processes in real time, when increasing humidity or exposing the particles to an atmospheric oxidant. By measuring the temperature, humidity, and pressure at the inlet and outlet of the holder, we can compare the identified deliquescence and efflorescence points with literature values. We can furthermore quantify growth rates and envision growth processes and crystallization pathways. Thus far we have investigated simple salts $(\mathrm{NaCl}$ seen in Figure 1), which we have exposed to air with an increasing humidity. Here we see the expected deliquescence when $\mathrm{NaCl}$ crystals take on water and dissolve as the humidity is increased. However, the setup also enables more complex scenarios, where multiphase particles or agglomerates are exposed to increasing humidity or oxidizing environments. Detailed knowledge of such processes can be fed into climate models to improve current predictions and assessments of climate change. The technique therefore has the potential to close crucial knowledge gaps in the current understanding of aerosol particles and their role in climate change.

\section{References:}

[1] Y Sato and K Suzuki, Science 363 (2019), p. 580.

[2] D Rosenfeld et al, Science 363 (2019), p. 80.

[3] O Boucher et al, Cambridge Univ. Press. 363 (2013), p. 571.

[4] JP Reid et al, Nat. Commun. 9 (2018), p. 1. 

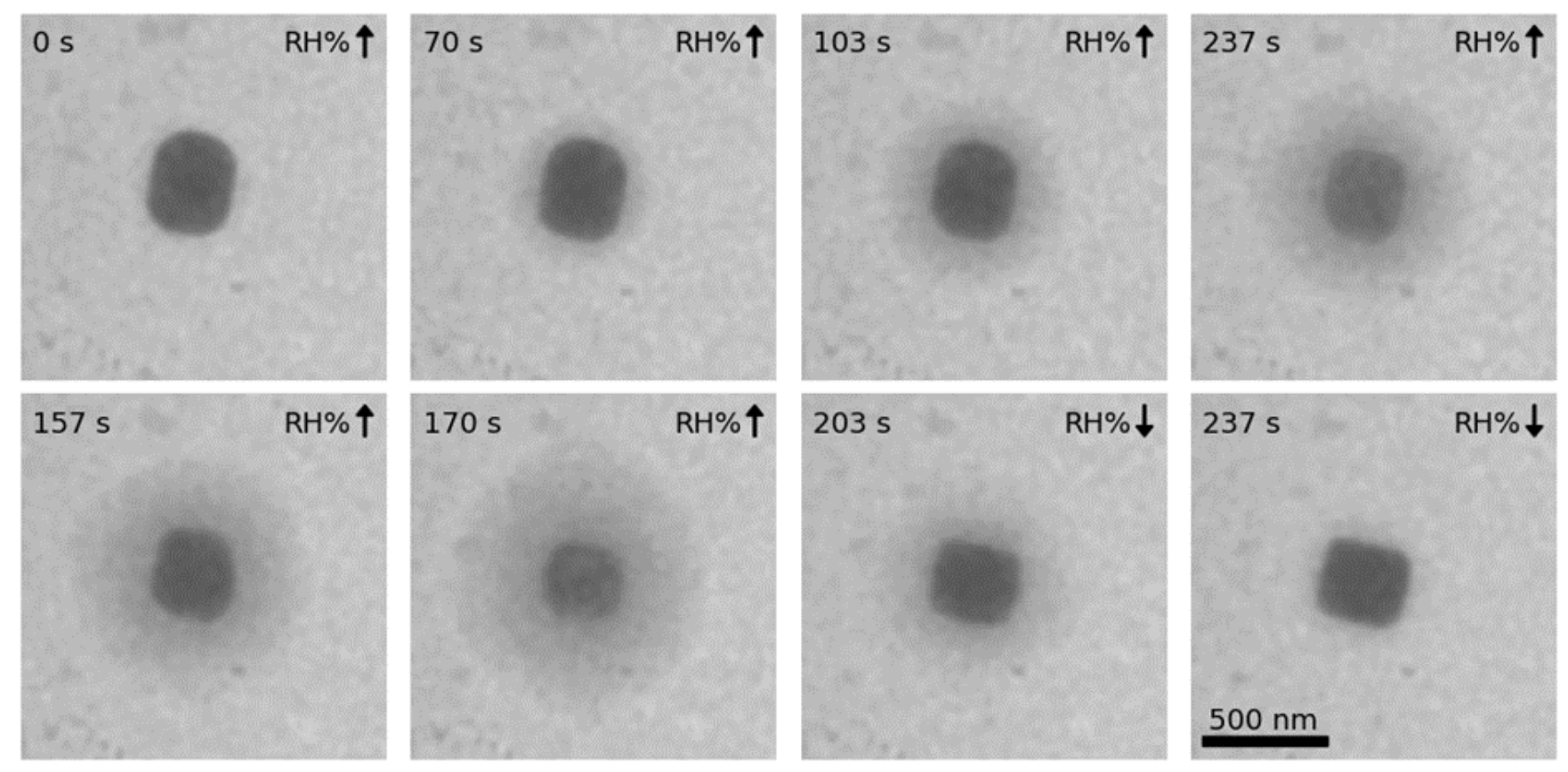

Figure 1. $\mathrm{NaCl}$ particle visualized inside the in situ holder under changing relative humidity. Humidity is increased in the first six frames and reduced in the final two. The scale bar is the same for all images, and a relative time is given in upper left corner. 